\title{
Expedition Inspiration Fund for Breast Cancer Research Meeting 2003
}

Harry Bartelink ${ }^{1}$, Christopher Benz ${ }^{2}$, Don Cleveland ${ }^{3}$, Ronald Dorn ${ }^{4}$, Julie Gralow ${ }^{5}$, William J. Gradishar ${ }^{6}$, Kathleen Grant ${ }^{7}$, Ruth Heimann ${ }^{8}$, Samuel Hellman ${ }^{8}$, Clifford Hudis ${ }^{9}$, Robert Kerbel $^{10}$, Marc Lippman ${ }^{11}$, John Lung ${ }^{4}$, Mitchell C. Posner ${ }^{8}$, Patricia Steeg ${ }^{12}$, Robert Vestal ${ }^{13}$, Ralph R. Weichselbaum ${ }^{8}$, and Bruce Zetter ${ }^{14}$

${ }^{1}$ The Netherlands Cancer Institute, Amsterdam, The Netherlands; ${ }^{2}$ Buck Institute for Age Research, Novato, CA; ${ }^{3}$ Ludwig Institute for Cancer Research, New York, NY; ${ }^{4}$ Mountain States Tumor Institute, Boise, ID; ${ }^{5}$ University of Washington, Seattle, WA; ${ }^{6}$ Northwestern University Medical School, Evanston, IL; ${ }^{7}$ California Pacific Medical Center, San Francisco, CA; ${ }^{8}$ The University of Chicago, Chicago, IL; ${ }^{9}$ Memorial Sloan-Kettering Cancer Center, New York, NY, USA; ${ }^{10}$ Sunnybrook \& Women's College Health Sciences Centre, North York, Ont., Canada; ${ }^{11}$ University of Michigan Health System, Ann Arbor, MI; ${ }^{12}$ National Cancer Institute, Bethesda, MD; ${ }^{13}$ Clinical Pharmacology Consulting; ${ }^{14}$ Harvard Medical School, Boston, MA, USA

The seventh Expedition Inspiration conference was held on February 19-23, 2003 at The Sun Valley Lodge. While there are other conferences that concentrate on a particular facet of breast cancer, the design and goals of this conference are unusual. In order to maximize interaction of investigators and clinicians the meetings are; limited in size, focused on presentation of current studies and allow time for extensive discussion. The participants include physicians and scientists involved in clinical and basic research as well as clinical practice. Some participants have been at previous Expedition Inspiration meetings while others are new. The meetings serve four purposes:

1. Stimulate discussion among participants who do not usually interact.

2. Develop consensus as to the state of our knowledge on particular aspects of breast cancer to stimulate future studies.

3. Develop collaborative projects among the meeting participants.

4. Foster new investigations by participants as well as others.

This year the topic was 'Metastasis'. We considered their biology and natural history as well as current and future therapeutic strategies. Our consensus follow:

\section{When in natural history does metastatic capacity develop?}

Fundamental to the prevention, diagnosis and management of breast cancer is determining when in the natural history of the disease the malignant potential is acquired. Within any clinically staged seemingly homogeneous group of tumors there is a continuum of tumors with different malignant potential extending form those tumors which, despite their small size and lack of lymph node metastases, have already developed metastases. Some of these have been detected in a study of gene expression profiles in individual tumors found in young women with clinically favorable disease. Since the 20-year disease-free survival of stage 1 breast cancer treated regionally is in excess of $80 \%$, the proportion of occult disseminated cancers within this group must be less than $20 \%$. At the other end of the spectrum are those tumors which if left untreated will continue to grow to very large volumes without developing metastases. Between these extremes are the majority of breast cancers. These appear to progress in their malignant potential during the clinical evolution of the cancer. This concept of tumor progression during the clinical phase of breast cancer is based on the correlation of tumor size with markers of malignant phenotype in a group of patients with very long follow-up. As tumor size increases the 
proportion of markers of proliferation, angiogenesis, invasion and distant spread also increases. Some of these phenotypes are acquired earlier than others but all demonstrate progression. For all tumors individual determination of their malignant potential is necessary to tailor management to the individual tumor's potential.

\section{Prognostic and predictive markers - new advances and future challenges}

Metastatic breast cancer remains a major cause of mortality among women in developed countries despite widespread breast cancer screening programs, improved regional therapy by surgery and radiation, and more frequent and aggressive use of systemic adjuvant therapy. Encouraging recent evidence of substantial declines in breast cancer mortality rates in several countries (despite increasing breast cancer incidence rates due to aging populations and increased screening) has come at the expense of overtreating many women diagnosed with early stage breast cancers, most of whom are offered toxic chemotherapy and/or years of endocrine therapy in order to save a few lives. After decades of searching for and evaluating the clinical usefulness of individual breast cancer biomarkers, clinicians are still unable to distinguish at diagnosis the $\sim 70 \%$ of node-negative breast cancer patients who are cured by regional therapy alone, yet must suffer acute and chronic side effects of additional systemic therapy, from the $\sim 30 \%$ of node-negative patients who clearly benefit from its lifesaving antitumor efficacy. Nonetheless, new advances from basic breast cancer research lend optimism to the expectation that individualized breast cancer therapy may yet be achievable.

We know that some individual biomarkers (e.g., MIB-1/Ki-67, ER/PR status) reflect the 'virulence' of a breast cancer at the time of its diagnosis but lack any long-term prognostic discriminatory value. Other markers better reflect the ultimate metastatic potential ('metastagenicity') of a primary breast cancer yet are limited by their prevalency or lack of prognostic accuracy. Some metastagenic markers are too commonly expressed among primary tumors to be clinically useful (e.g., uPA, angiogenic markers), although their absence may identify a small fraction of patients with high likelihood of remaining disease-free for 10 or more years after primary therapy and without need for any adjuvant therapy. In contrast, other known mark- ers of metastagenicity are found too infrequently in early stage primary breast tumors and likely arise with advancing breast cancer stage or during its clinical progression. (e.g., p53 mutation/overexpression, loss of $\mathrm{nm} 23$ or E-cadherin expression).

A weak prognostic marker like estrogen receptor (ER) status may still have important clinical value as a predictive marker, identifying patients most likely to benefit from adjuvant endocrine therapy with either an antiestrogen or aromatase inhibitor; and multiple lines of new evidence now point to the existence of clinically relevant subsets of ER-positive breast cancers. Likewise, we expect further clinical subsetting of breast cancers presently lumped into one category by the predictive marker, ErbB2 receptor amplification/overexpression. New immunohistochemical reagents capable of identifying molecular pathway differences among ER-positive and ErbB2-positive breast cancer groups are being evaluated and will be critical in defining new targets and designing more effective and individualized ER- and ErbB2-specific therapeutics.

Most encouraging are the novel bioengineering advances enabling various researcher to probe freshly harvested breast cancers and simultaneously quantitate in each the expression of all known human genes $(\sim 30,000)$, arrayed on the surface of a single microscope slide. Many such microarray studies have been reported in the past 2 years; and several landmark reports have proven that breast cancers can be classified into clinically relevant subsets previously indistinguishable by pathologic evaluation, clinical staging, or standard biomarker studies. It now appears that informative groups of as few as 70 different genes can be used to 'cluster' breast cancers into prognostically different subsets at the time of diagnosis, and potentially classify individual patients according to their risk of developing metastatic disease and thus determine their need for systemic adjuvant therapy. Curiously, only a few of our formerly validated biomarkers have emerged in these prognostic gene 'signatures'. Once the reproducibility and accuracy of standardized gene signature sets have been clinically validated, future challenges will face both basic and clinical breast cancer researchers who will need to decipher the biological roles played by these genomic signatures, according to their individual protein functions and tumorigenic pathways. To understand the biological and clinical import of all this gene expression data, we certainly anticipate the need for new tools and technical 
advances emanating from the much younger field of proteomics.

\section{The development and validation of new therapeutic targets}

The remarkable growth in our understanding of the cellular molecular biology of breast cancer should result in the identification of potential new targets for therapy. Several of these have already reached the clinic including the targeting of the epidermal growth factor receptor (i.e., Iressa, Tarceva). Many trials are currently underway attempting to validate a variety of anti-angiogenic targets as well. In the near future there will be an increasing focus on other potential pathways, which differentiate the growth and survival of breast cancer from normal cells including the targeting of telomerase as well apoptotic pathways. The latter appears particularly promising since overexpression of anti-apoptotic molecules has been widely documented in human breast cancer and preliminary pre-clinical data suggest that inactivation of anti-apoptotic molecules not only provides therapeutic gain, but dramatically enhances the effectiveness of more traditional cytotoxic chemotherapies and radiation treatment.

We believe that for such approaches to rapidly be developed in a clinic a variety of avenues need to be more effectively exploited. These include the following: First, it is essential that clinical trials be designed in such a fashion that sufficient clinically derived material is available both before and preferably after therapeutic interventions to allow sophisticated analysis of target validation. Clearly the ability to perform expression arrays, comparative genome hybridization analysis, proteomic analysis, etc. require a considerable effort to obtain such material from patients. It is already the case that many clinical trials have demonstrated activity in only small sub-sets of patients. Unfortunately this may lead to the abandonment of an otherwise promising agent as a result of the failure to appreciate that subtle differences in the biology of different cancers that could identify patients likely to respond. Better analysis of interactions with tumor targets could prove that these agents have considerable clinical value. In this same context, more widespread exploitation of so-called 'warm' autopsies would allow an analyses of multiple metastatic sites. A wealth of potential information concerning heterogeneity as well as the cellular molecular biology of tissue specific metastases could result in more effective use of novel biological therapies. ACTION ITEM: Without investment in detailed analyses of tumor samples efficient development of novel biologic agents will be greatly hampered.

\section{Role of accessory cells in tumor progression and metastasis}

It is well documented that tumors are comprised of a variety of normal cells that are recruited to the tumor site. These cells include vascular endothelial cells and pericytes, inflammatory cells such as neutrophils, monocytes and macrophages, as well as lymphocytes and fibroblasts. In breast cancer, metastases to bone encounter large number of bone cells (osteoblasts and osteolclasts) as well as hematopoietic stem cells in the bone marrow. These various stromal cell populations provide an opportunity for therapeutic interventions that target a population that is less mutable than the tumor cells themselves and may consequently be less susceptible to developing drug resistant subpopulations.

The best characterized agents that target the stromal cell compartment are anti-angiogenic drugs that antagonize vascular endothelial cells. Many recently developed agents target the vascular endothelial growth factor (VEGF) either directly or via its receptors. The inability of bevacizumab (Avastin) to enhance the clinical outcome of patients with metastatic breast cancer treated with capecitabine, may reflect flaws in the strategy of optimally utilizing anti-angiogenic agents rather than a failure of the antiangiogenic strategy for cancer treatment. Angiogenesis inhibitors may be used successfully in treating breast cancer especially when used in conjunction with other therapies.

It has also been possible to use conventional cytoxic agents in low-dose, long-term 'metronomic' schedules. When used in this way, these frequently administered agents exhibit anti-angiogenic activity and may cause extended tumor stabilization in the absence of severe dose-limiting toxicities. In this regard, there are a number of low-dose metronomic chemotherapy trials that are underway in the US, Canada and Europe that involve both adjuvant and metastatic therapy of breast cancer. These trials include protocols such as a weekly taxane combined with Avastin, daily oral cyclophosphamide and twice weekly oral methotrexate either alone or in combination with a drug such as avastin or thalidomide. It has been show that low-dose 
regimens are sometimes effective even when resistance has been developed to higher doses of the same drug.

Recent genomics studies have revealed that metastatic cells in a variety of cancers, including breast cancer, have a characteristic 'signature' of genes whose levels are positively or negatively regulated in both the primary tumors and metastatic colonies. Intriguingly, many of these are suggested to be derived from stromal cells rather than from the tumor cells themselves. These results indicate that one hallmark of the metastatic tumor is the ability to attract and activate accessory cells. For example, tumor-associated fibroblasts may interact with tumor cells via paracrine pathways involving IGF-1 and PDGF and their receptors. Consequently, drugs such as STI571/Gleevec may have some utility in disrupting such interactions because of its ability to block PDGF receptor signaling. Thus tumor-associated cells, along with their attractants and activators may represent the next critical target for novel adjuvant therapies that will complement and potentiate the efficacy of conventional therapeutic agents.

\section{Genetic control of metastasis}

While many molecular alterations induce or promote the metastatic behavior of tumor cells, other genes have been found to suppress this activity. To date, four metastasis suppressor genes have been confirmed in breast cancer model systems, nm23, Brms1, KAIl and Kiss1. Each, when transfected into a metastatic breast carcinoma cell line, reduced in vivo metastasis without appreciable affects on tumorigenicity. Other metastasis suppressor genes have been identified and await testing in breast cancer. Metastasis suppressors exhibit novel functions which have highlighted new aspects of the metastatic process, such as differentiation, growth factor receptor down-regulation, neuropeptide signaling and gap junctional communication. Most metastasis suppressor genes are not mutated, but simply down-regulated at the transcriptional level. It is hypothesized that re-expression of these genes in micrometastatic breast cancer cells may limit their subsequent colonization and invasion, and induce differentiation, with a potential clinical benefit.

Another incompletely defined aspect of metastasis is the effect of host genotype on metastatic spread. The set of genes that we are born with may influence the propensity of tumors to metastasize. For example, a transgenic which develops metastatic mammary cancer can be bred to various other strains of mice; F1 generations all express the same mammary transgene but differ 100-fold in metastatic efficiency. The identification of host genes which influence metastatic efficiency is a high priority, and could lead to metastasis preventative strategies for those at high risk.

\section{Need for developing and using better preclinical models of breast cancer metastasis}

There is a need to develop better mouse models of breast cancer metastatic disease to use for testing promising new drugs and treatments. In this regard, there is a wide-spread perception that currently used preclinical models, including breast cancer, are of extremely limited value in predicting the effects of new drugs and treatment strategies when these are moved into phase I, II, or III clinical trials. However, such clinical trials mostly involve patients with advanced high-volume metastatic disease, sometimes involving sites such as the brain or bones, in addition to the liver and lungs. In addition, patients in clinical trials have often been heavily pretreated with standard therapies prior to recruitment into a clinical trial and as such, are generally less responsive, that is, more resistant, to subsequent treatments. It is extremely rare to initiate treatment in preclinical models such as human tumor xenografts or spontaneously arising tumors in transgenic/knockout mouse model of cancer when metastatic disease is highly advanced, and/or drug resistant. In retrospect, treatment is generally initiated on low-volume, minimum residual metastatic disease, that is treatment naive. This could explain the disparity that is frequently observed between the results obtained in such preclinical mouse models and clinical trials.

More emphasis should be placed on preclinical models involving advanced, high-volume metastatic disease, including drug resistant tumors. Such studies are particularly timely because of various technological advancements enabling quantitative detection in changes of metastatic disease burden. These advances include the application of non-invasive small animal functional imaging (e.g., microPET and microMRI), the use of fluorescent tagged tumor cells and the use of tumor cells genetically engineered to secrete tumor-specific protein markers that can be detected in serum, plasma or urine using ELISA assays. Needed 
are spontaneously arising mouse breast cancers which metastasize more aggressively and to clinically relevant sites. For example, bone and brain metastases are exceptionally rare in both human tumor xenografts or spontaneously arising mouse breast tumors. Since CNS metastases may become of greater importance over the next decade as a result of successes in treating metastatic disease in other sites with new drugs, greater effort should be placed on trying to develop models of brain metastases in mice with human or mouse breast cancer. A similar emphasis should be placed on improving models of bone metastasis.

\section{Incrementalism matters}

While society appropriately seeks dramatic breakthroughs in the effort to cure breast cancer, it is important to recognize that a series of modest incremental improvements in outcomes can offer significant overall benefits and has already done so. For a disease like breast cancer where there does not appear to be a precise and consistent lesion - breast cancer is a collection of related diseases but is not one illness with one consistent genetic defect - a single new drug or intervention seems unlikely to offer a breakthrough such as has been seen in a few selected illnesses. Examples of diseases that are caused by a single or limited number of genetic defects where a specific intervention (drug) has offered a dramatic change in outcomes, include APL treated with retinoids and CML or GIST treated with Gleevec. Yet in the case of Acute Lymphocytic Leukemia, similar changes in natural history have been achieved through a series of improved conventional treatments. Over several decades a series of randomized clinical trials, broadly supported by the pediatric community, has yielded a dramatic improvement in survival for affected children. This is an important lesson for us as we approach the problem of breast cancer. Over the past several decades we have incrementally added hormonal therapy, a variety of chemotherapy agents, and more recently a targeted antibody approach (trastuzumab) and have seen clinical trials yielding a series of sequential modest improvements contributing to a remarkable fall in the absolute number of deaths from breast cancer over the past decade even as the population ages and the incidence has remained constant or possibly has increased. The inescapable conclusion is that while each individual intervention provides only a small benefit, the aggregate of a series of small benefits can be far more significant. Hence, we must recognize the clinical importance of very modest benefits, particularly in a common disease, and we call for broad support of appropriate randomized clinical trials that offer the potential to uncover more of these small but important incremental improvements.

\section{Pharmacogenomics}

In recent years there has been great interest in developing agents that target pathways critical to the growth and survival of human cancer. Underlying this approach has been the reasonable assumption that understanding the diverse and heterogeneous pathways by which cancers grow can provide new approaches to the treatment of the disease. Relatively less attention has been given to the area of pharmacogenomics. It is clear, however, that in many circumstances therapies may fail not because of their theoretical lack of effectiveness, but simply because either adequate concentrations of drugs are not made available to the tumor or an unappreciated toxicity develops.

At least $85 \%$ of all drugs administered to human beings are either activated or metabolized by a series of enzymes (largely the cytochrome P450 family found in the liver). Substantial genetic heterogeneity exists in the human population for most of these genes. It has now become possible to provide sequence information on most of the P450s and therefore predict likelihood of toxicity. In addition, a series of experimental approaches in which test dose of drugs metabolized by similar pathways or can be analyzed and rational dosing schedules developed exactly as drug doses are commonly modified for body surface cells and renal function. It is already clear that in the case of such commonly used agents as tamoxifen, cyclophosphamide, venolrelbine and paclitaxel that substantial genetically based variations in cytochrome $\mathrm{P} 450$ s can lead to dramatic differences in effective dosing. Unfortunately, this information has never been tested prospectively in clinical trial design. It is certainly the case that in many situations failure to observe objective responses to therapy is due less to the intrinsic lack of activity of the agent in question but rather the failure to achieve effective dosing.

We propose as an ACTION ITEM that substantial attention be given to pharmacogenomic analysis both of existing and newly developed agents so that they may be used rationally and with maximal effectiveness in clinical trials. 


\section{Regional therapeutic strategies for metastatic disease}

Regional treatment strategies for patients with disseminated breast cancer directed at sites of residual or large volume disease are a potential therapeutic approach that deserves study in the context of improving outcome of patients treated with systemic therapy. Regional approaches, including metastasectomy and tumor ablation, are routine and effective treatment options in the management of disseminated colorectal cancer and soft tissue sarcomas. These same techniques have been applied to other tumor types, including breast cancer, on a much more limited basis in carefully selected patients with varying degrees of success. However, these techniques are not considered standard treatment for metastatic breast cancer. The hypothesis for successful regional treatment of metastatic disease is that the metastatic phenotype has not been fully expressed as evidenced by metastases that are limited in number and restricted in location. The mechanisms responsible for such oligometastases has not been fully elucidated but may involve factors that include host, tumor cell, and stroma that predispose to an indolent and favorable biologic behavior. Furthermore, it is not unreasonable to presume that as more effective systemic therapy is introduced, the natural history of breast cancer may be altered and dominant sites of metastases may emerge. Even if metastatic sites are not exclusive, they may have a predominant impact on clinical behavior/survival and therefore, directing therapy to these sites may substantially affect outcome. For example, hepatic metastases are rarely isolated in patients with breast cancer, however, approximately $50-75 \%$ of patients with disseminated disease have liver involvement, which results in death in a significant percentage of patients. Therapeutic approaches directed toward metastatic deposits in the liver, bone, or brain, if effective, could result in improvement in both quality and quantity of life.

\section{Regional approaches to metastasis}

Gene therapy of cancer involves tumor-specific delivery and expression of genes encoding proteins that are directly or indirectly toxic to tumor cells or tumor stroma. Strategies to enhance specificity of tumor targeting and tumor-specific control of therapeutic gene expression include physical and transcriptional targeting. Promoters such as Egr-1 and MDR1 have been ligated upstream of therapeutic transgenes in viral vectors that are injected intratumorally. These promoters can be activated by ionizing radiation and/or chemotherapy driving transcription of therapeutic genes, leading to an enhanced antitumor response demonstrated in preclinical tumor models. This system allows both spatial and temporal control of gene therapy to the tumor volume. Tissue or tumor selective promoters to activate transcription of therapeutic genes to enhance and target gene therapy are also being explored. For example, adenocarcinomas that overexpress DF3/MUC1 (overexpressed in approximately $80 \%$ of human breast carcinomas) will preferentially express therapeutic genes that are transcriptionally activated by the MUC1 promoter. Strategies are currently under investigation to deliver these viral constructs via regional arterial routes, as well as, physically altering virus' to enhance tumor tropism and allow systemic delivery of these vectors. Combining this type of regional therapy with systemic therapy is one of many models that require further study in an attempt to improve the treatment and eventual outcome of patients with disseminated breast cancer.

Address for offprints and correspondence: Samuel Hellman, MD, The University of Chicago, 5758 S. Maryland Ave., MC 9006, Chicago, IL 60637, USA; Tel.: +1-773-702-4346; Fax: +1-773-702-4347; E-mail: s-hellman@uchicago.edu 